\title{
Performance Evaluation of AODV and OLSR for Different Mobility Patterns By Varying Source Nodes in MANET
}

\author{
Anuradha ${ }^{1}$, Inderjeet Singh Gill ${ }^{2}$ \\ Research Scholar, SBSSTC, Ferozepur, India ${ }^{1}$ \\ Assistant Professor, SBSSTC, Ferozepur, India ${ }^{2}$
}

\begin{abstract}
MANET is a network of wireless mobile nodes that exchanges information forming a self-governing and self-motivated network without any central management and fixed infrastructure. There is always a requirement of energy efficient and high speed protocols to find a path for forwarding data packets through relay nodes to the destination node. The selection of mobility pattern with high degree of realism is also an important characteristic used for evaluating the performance of wireless network. In this research paper the performance evaluation of wireless network using existing mobility models: Manhattan Grid Mobility Model (MGMM) and Random Waypoint Mobility Model (RWPM) is made and a new such model is proposed combining the features of existing models. The comparison is then made by using AODV and OLSR as the routing protocols and the functionality is analyzed in terms of different parameters: throughput, data drop and traffic received. The preliminary performance demonstrates that the proposed mobility model is found very close to MGMM and better than RWPM in terms of data drop and traffic received.
\end{abstract}

Keywords: MANET, AODV, OLSR, Mobility Patterns, Traffic Models.

\section{INTRODUCTION}

Wireless communication has become the essential part of communication in today's era as it provides an ease to the users to communicate anywhere even while travelling. Mobile Adhoc Network [MANET] which is one of the subareas of wireless networks [4] has become the good choice for the researchers to explore and perform simulation analysis by considering various mobility models and traffic patterns $[1,2]$. MANET is a network of wireless mobile nodes that exchanges information forming a self-governing and self-motivated network without any central management and fixed infrastructure. There are numerous approaches to communicate wirelessly like WiMax, Bluetooth, WI-Fi and zigbee etc. These all systems give short range communication. However, in the events like in military field, earthquake relief, and other areas of the emergency communications there is always a need for long distance wireless communication and it is there MANET plays significant role. In MANET since there is movement of nodes and due to this movement design of energy efficient protocols has become a challenging issue for the researchers [1]. Due to high mobility of nodes, security is also a major issue and it is found that the communication done using MANET is highly secure. Due to its high security feature it is being used in numerous applications like military applications for robotized combat zone, exceptional operations, country guard, and rescue areas etc. Some other areas include natural disaster recuperation (rush, fire, earth quake), law enforcement (crowd control), environment monitoring (sensors) and space/planet investigation [7, 9].

There is different mobility models used in analyzing the performance of MANET used for different applications. Few of them are: Random Way Point, Manhattan Grid
Mobility Model, Reference Point Group Mobility Model (RPGMM), Freeway Mobility Model, Gauss Markov Mobility Model etc that have been used for evaluation. RWMP and MGMP are commonly used for evaluating the performance of the network. In this research paper OLSR and AODV protocols are analyzed in terms of throughput, data drop and traffic received by using MGM (Manhattan grid mobility)[17], RWP(random way point mobility) and a new mobility pattern developed in this research named active inactive mobility pattern which is compared with existing patterns in terms of various parameters.

\section{ROUTING PROTOCOL}

An ad-hoc routing protocol is a set of rules which are standardized and justify how nodes choose the path to send data packets in between the communicating devices in a mobile ad hoc network system. The distinctive protocols are used to manage steering or routing issue in the MANET [1, 2, 3]. These routing protocols are classified into three categories that are Reactive, Proactive and Hybrid [5, 9]

A. Reactive (On demand) routing protocols

This is otherwise called on-demand routing and ondemand driven reactive protocols. They don't launch route disclosure independent from anyone else, until they are asked for, route is just found at whatever point they are really required [12].

One such reactive protocol being used is AODV.

- Ad- Hoc on Demand Distance Vector (AODV) Protocol

AODV is an example of reactive protocol and it gives path on demand. It mainly manages three important messages that are RREQ (Route Request), RREP (Route Response) 
and RERR (Route Error). AODV is the advance version of DSR (dynamic source routing) as DSR includes source routes in packets header resulting in large header which degrade the performance of the network. AODV is an attempt to improve on DSR by maintaining routing tables at the nodes, so that data packets do not have to contain routes. It attains the desirable features of DSR that routes are maintained only between nodes which need to communicate $[2,3]$.

\section{B. Proactive (Table driven) routing protocol}

In proactive protocol the routes are maintained each and every time from one node to all other nodes. Route formation and preservation is done through some arrangement of periodic and event-triggered routing updates [13]. The updates happen at particular intervals, in spite of the mobility and traffic description of the network system. At whatever point there is revolutionize in the system topology, these tables are repaired by change [14]. One such protocol being used is OLSR.

- $\quad$ Optimized Link State Routing (OLSR) Protocol The most suitable example of proactive or table maintained link-state routing protocol is Optimized Link State Routing (OLSR) Protocol [8]. As it is proactive in nature, its advantage is that routes are immediately available whenever needed, because all the nodes are connected to each other all the time by maintained routes.

This protocol overcomes the drawback of AODV in which flooding of request messages occurs in the entire network but in OLSR protocol optimization of AODV protocol is carried out. Firstly the size of control packets is reduced and secondly it minimizes flooding of control traffic by making use of the selected nodes to transmit its messages to the network. Even in case of link failure it does not generate the extra control traffic. OLSR is mainly suitable for large and dense networks and where communication between the nodes is quite frequent. As this protocol is not reliable so there could be loss of packets from time to time. There is no need to sequence the order of delivered packet as every packet has a sequence number in this protocol [6].

\section{MOBILITY PATTERNS}

It describes how nodes can move in a specific scenario or area. From previous work in this field it is concluded that different mobility models could lead to variation in the performance of protocol. Different parameters like throughput, overhead, data drop, delay etc. of a protocol can vary extensively when used with different mobility models. The performance of network degrades when simulation is carried out with inappropriate mobility model. Relative performance of the protocol also gets affected with the model.

For example, throughput observed in results is larger when MGM is used with AODV as compared to when MGM is used with OLSR [22].

\section{A. Manhattan Mobility Model (MM)}

The main motive for introduction of Manhattan mobility was to imitate the movement pattern of mobile nodes on vertical and horizontal streets defined by maps. Each street is further divided into two different lanes in north \& south direction for vertical streets and east \& west in case of horizontal streets. The mobile node is meant to move beside the grid of vertical and horizontal streets on the map. Figure 1 show the movement traces of mobile nodes in Manhattan MM [21].

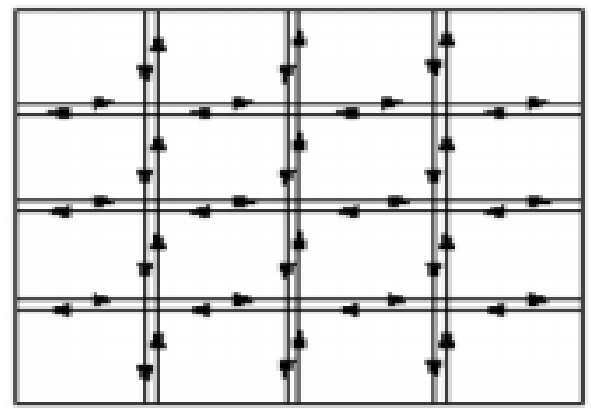

Fig 1: Movement pattern of mobile nodes in Manhattan MM [21]

B. Random way point mobility model (RWPMM) RWPMM is an entity mobility model and was first used by Johnson and Lee [21]. It included pause times between changes in direction and speed. Mobile nodes are placed to move randomly in any direction within simulation area. Each mobile node is free to select its direction, speed, destination independent of the neighboring nodes. This model is widely implemented and analyzed in simulation of routing protocols due to simplicity and availability.

\section{Active Inactive mobility model}

Degree of realism is an important requirement of any model in actual world. More real models would lead to more accurate simulations and evaluation of network performance parameters. In this proposed active inactive mobility model few nodes are considered as source node and others as the relay node, both of which could either are stationary or mobile. In this model both the mobility models i.e. MGMM and RWPMM using two traffic models namely exponential and Poisson are used with AODV and OLSR routing protocols and the performance is analyzed.

\section{RELATED WORK}

Even though lot of research has been done in this area but there are still various research issues which are yet to be dealt on. Sunil Kumar Singh, Rajesh Duvvure, Amit Bhattcharjee [17] analyzed the effect of different mobility models on different protocols like OLSR, TORA and zone routing protocol with use of CBR traffic and concluded that a few mobility models performs better in different routing protocols. Arindrajit Pal, Jyoti Prakash Singh[18], presented the behaviour of the mobile nodes for different speed for three different traffic patterns such as CBR, Exponential and Pareto. They found through simulations that the AODV routing protocol performs much better than DSR in Exponential and Pareto traffic. They also analyzed the performance parameters for different traffic patterns. Anuj K. Gupta, Harsh Sadawati [21] conducted a survey on mobility model and stated the importance of mobility model in the accurate simulations 
of network. Dimitra Kampitaki, Anastasior A. Economides [25] stated that the performance and operation of the most popular routing protocols used in MANET is affected by the type of traffic load in the network regardless of mobility model being used.

\section{V.CASE SCENARIO}

In this paper the performance analysis of ADOV and OLSR protocol is analyzed under different mobility patterns (Random way point, Manhattan Grid Mobility Model and Active Inactive mobility) using FTP application. The nodes are randomly placed over the area of $1000 \mathrm{~m} * 1000 \mathrm{~m}$. To analyze this performance different cases are taken using OPNET modeler 14.5 [8].

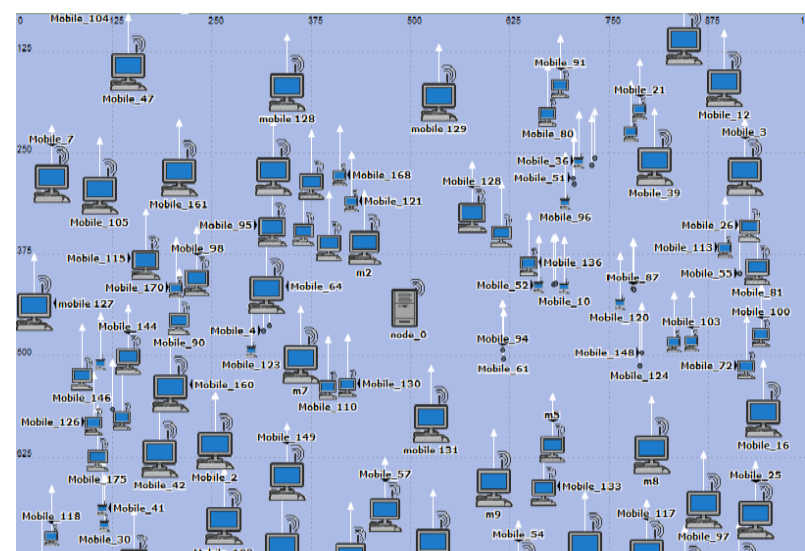

Fig 2: Simulation Environment

Firstly simulation is done using Exponential traffic then Poisson traffic is used. In total 129 nodes are taken out of which initially 10 nodes are taken as source nodes and rest of other work as relay nodes while in another case 5 nodes are taken as source nodes and remaining as relay nodes between source and destination. In both cases different mobility patterns are applied to relay nodes and source nodes by making different scenarios. These cases are made firstly by using OLSR and then by using AODV and performance analysis is made.

\section{TABLE 1: SIMULATION PARAMETERS}

\begin{tabular}{|l|l|}
\hline $\begin{array}{l}\text { Network } \\
\text { Parameters }\end{array}$ & Values \\
\hline $\begin{array}{l}\text { Number of FTP } \\
\text { source Nodes }\end{array}$ & 10 (case I), 5(case II) \\
\hline Simulation Time & $60 \mathrm{sec}(1 \mathrm{~min})$ \\
\hline Simulation Area & $1000 \mathrm{~m} \mathrm{X} \mathrm{1000} \mathrm{m}$ \\
\hline Routing Protocols & AODV, OLSR \\
\hline $\begin{array}{l}\text { Node Movement } \\
\text { Model }\end{array}$ & $\begin{array}{l}\text { Random Waypoint, Manhattan } \\
\text { mobility model, active inactive } \\
\text { model. }\end{array}$ \\
\hline Data Rate & $24 \mathrm{mbps}$ \\
\hline Application Name & FTP \\
\hline Simulator & Opnet Modeler 14.5 \\
\hline Relay node speed & $7 \mathrm{~m} / \mathrm{s}$ \\
\hline Packet size & $2 \mathrm{MB}$ \\
\hline
\end{tabular}

\section{RESULTS}

The main objective of this research paper is to analyze the performance AODV and OLSR protocols by varying the number of source nodes for FTP application. The result is taken in terms of Throughput, Data Dropped and Traffic received.

A. Throughput

It is the total number of bits that are forwarded from wireless LAN layers to higher layers in all WLAN nodes of the network, and is measured in bits/sec [19]. All the four graphs of throughput shows time span of 60 seconds along $\mathrm{x}$-axis and throughput measured in bits/sec along $\mathrm{y}$ axis. The throughput comparison done between OLSR and AODV protocols for 10 and 5 source nodes shows that MGM has highest throughput followed by active and inactive model with very less difference and proposed model gives better throughput than RWP.

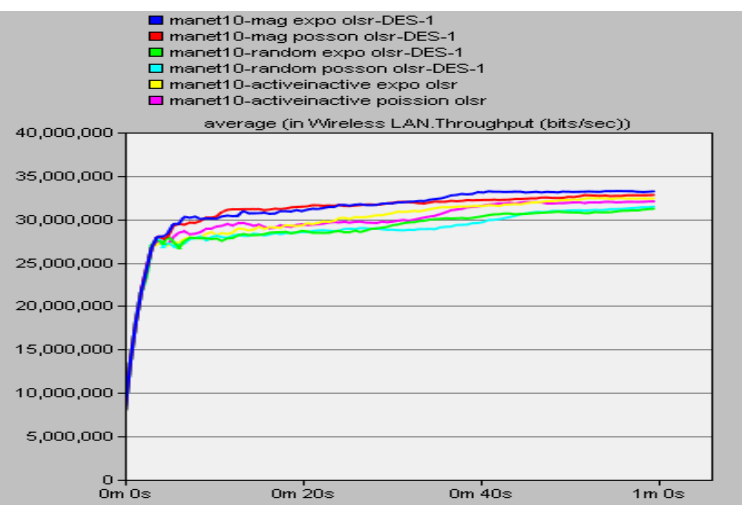

Fig 3: Throughput of 10 source nodes for OLSR protocol

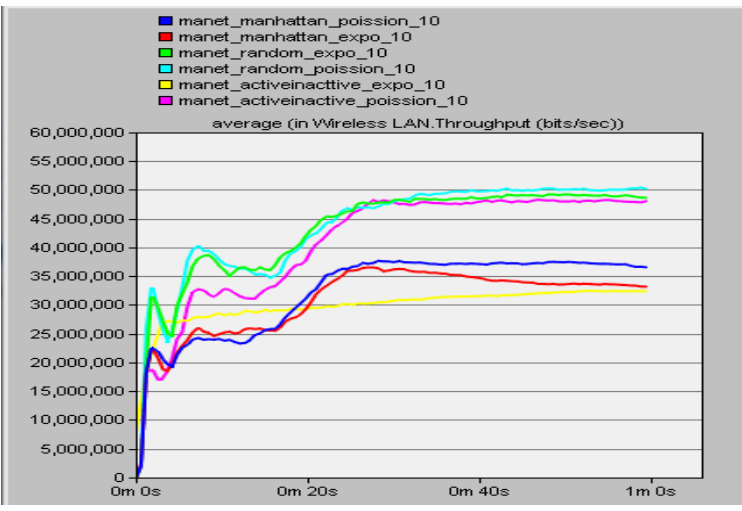

Figure 4: Throughput of 10 source nodes for AODV protocol

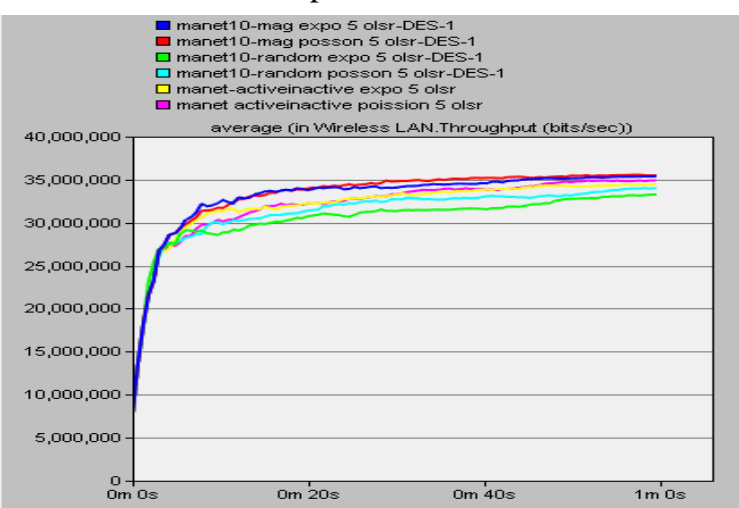

Figure5 Throughput for 5 source nodes for OLSR protocol 


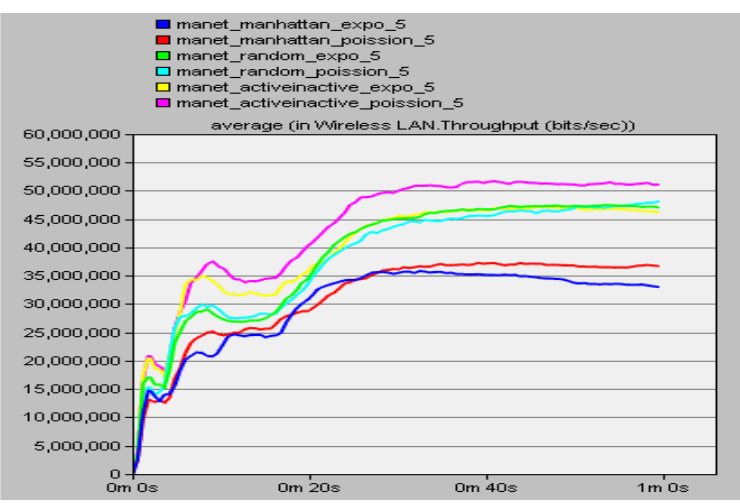

Fig 6: Throughput of 5 source nodes for AODV protocol

B) Data Drop

This statistics represent the total higher layer data traffic (in bits/sec) dropped by the all WLAN in the network as a result of consistency falling retransmission [20].

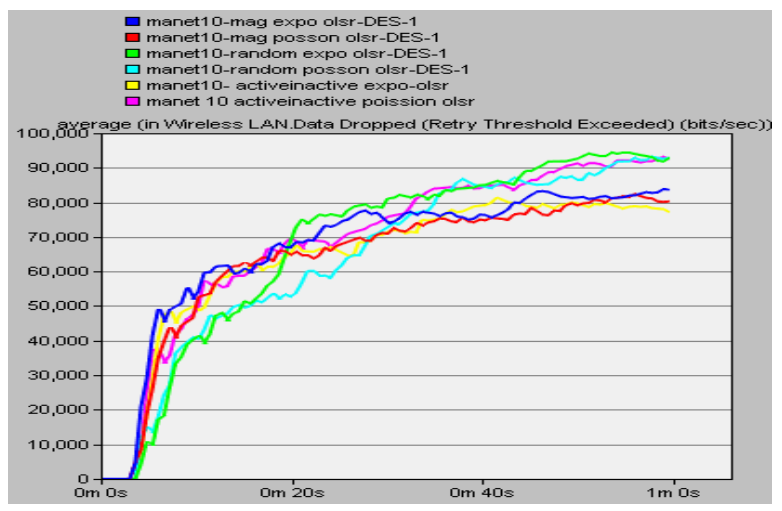

Fig 7: Data Drop for 10 source nodes for OLSR protocol

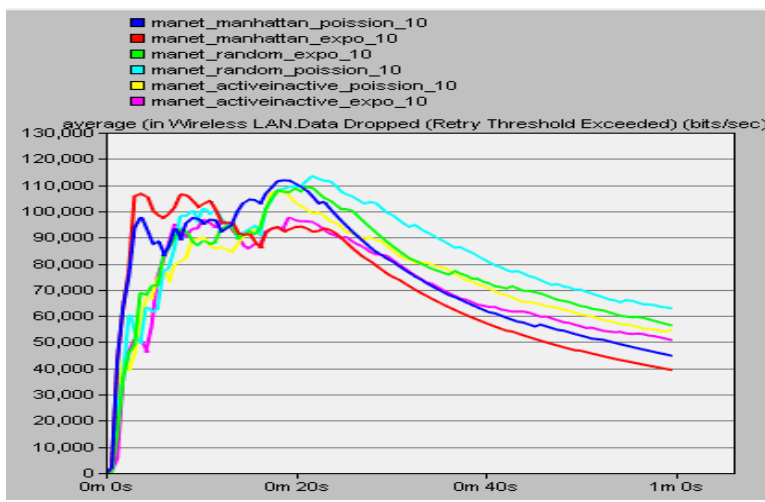

Fig 8: Data Drop for 10 source nodes for AODV protocol

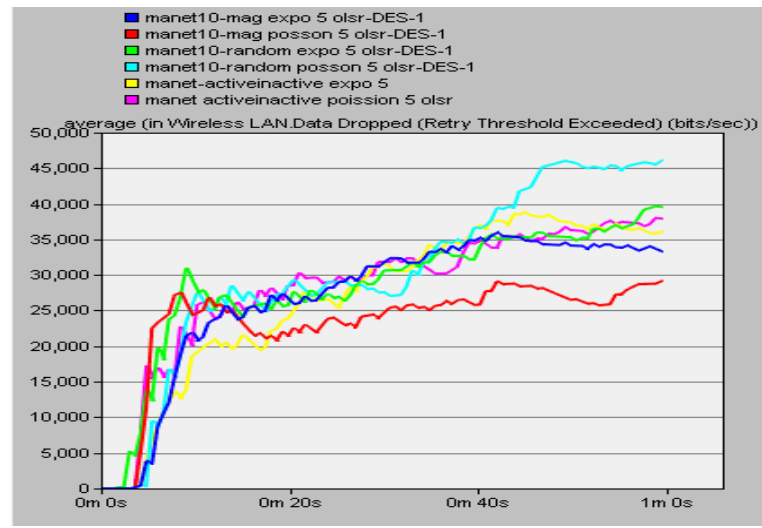

Fig 9: Data Drop for 5 source nodes for OLSR protocol
Along all the four graphs of data drop $60 \mathrm{sec}$ time span is taken along $\mathrm{x}$-axis and data drop measured in bits/sec is taken along y-axis. From below figures it could be concluded that for both cases active inactive mobility performs very close to MGM.

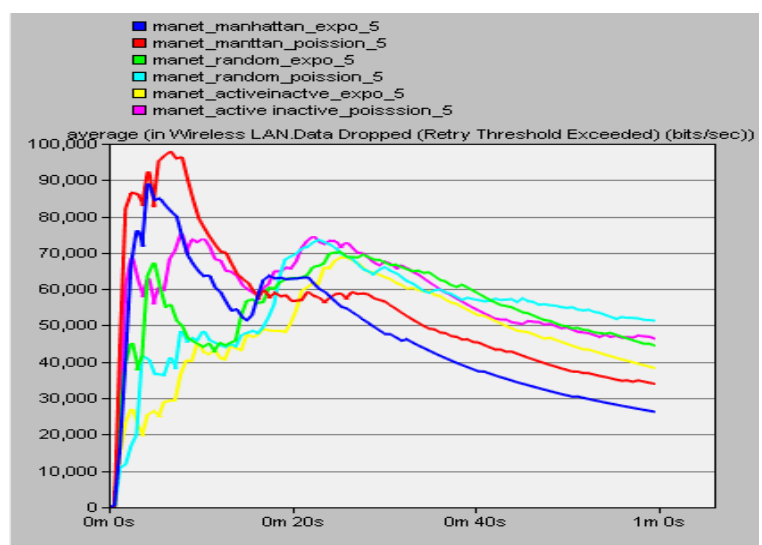

Fig 10: Data Drop for 5 source nodes for AODV protocol

\section{C) TRAFFIC RECEIVED}

Traffic received is average bytes per second forwarded to all FTP application by the transport layers in the network. Traffic received increases as the number of source nodes increases. From below figures it is observed that traffic received in case of active inactive mobility is far greater than the RWP.

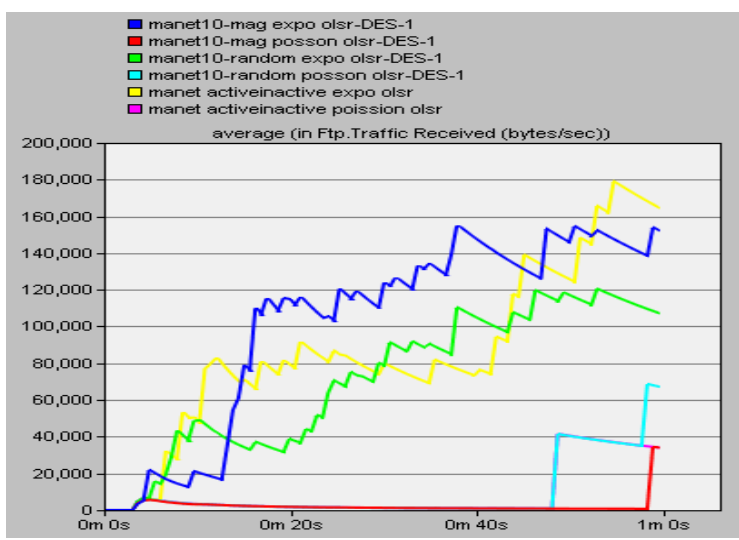

Fig11: Traffic received for 10 source nodes for OLSR protocol

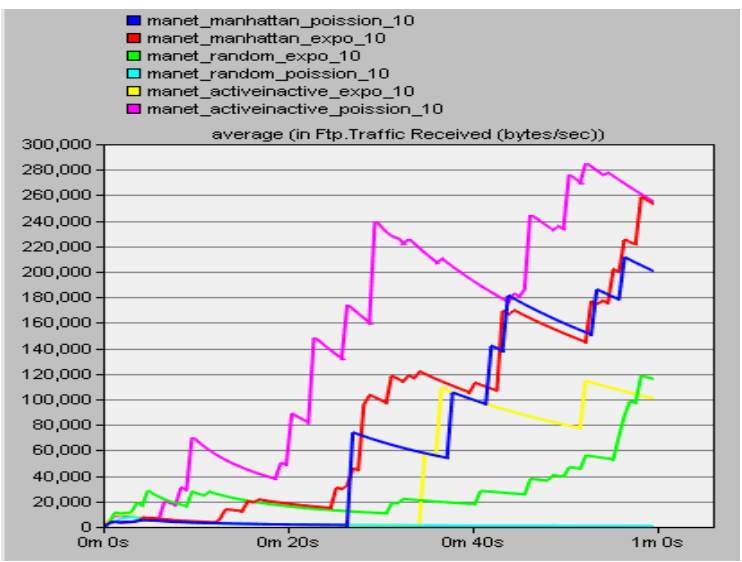

Fig.12 Traffic received for 10 source nodes for AODV protocol. 
In the following graphs $60 \mathrm{sec}$ time span is taken along $\mathrm{x}$ - Inactive mobility models. The simulation of AODV and axis and traffic received measured in bytes/sec is taken along y-axis.

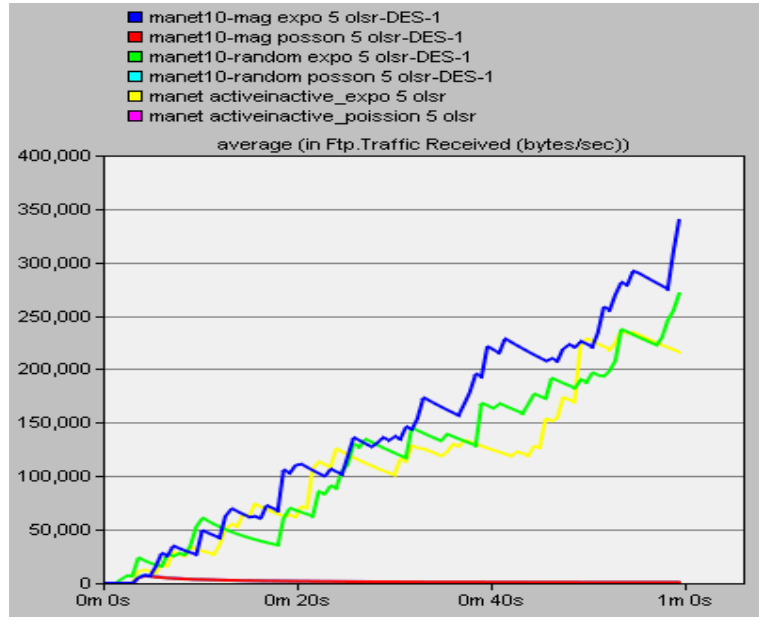

Fig13. Traffic received for 5 source nodes for OLSR protocol

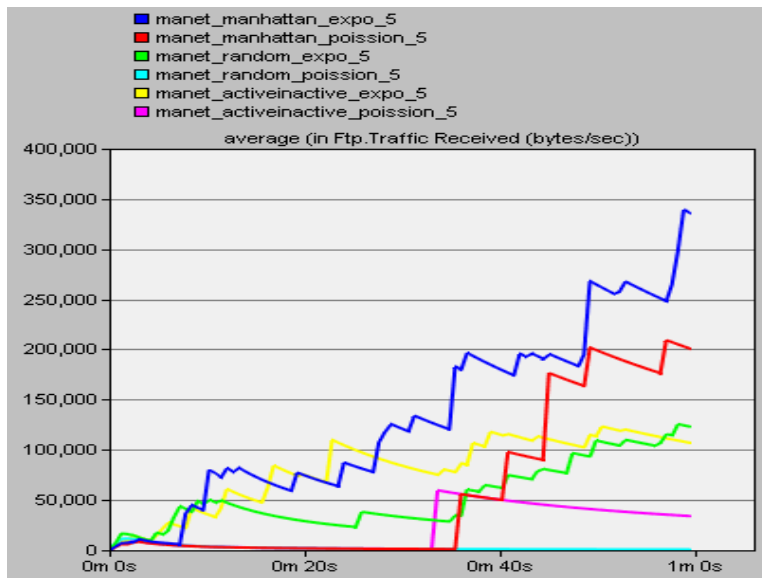

Fig: 14 Traffic received for 5 source nodes for AODV protocol

\section{CONCLUSION}

In this paper the performance of AODV and OLSR is compared by varying source nodes. This comparison is done by using Exponential and Poisson traffic models. For this simulation 125 nodes are used among which some nodes are relay nodes which are used between source and destination nodes. The placement of nodes is random over the area of $1000 * 1000 \mathrm{~m}$. The result shows that Manhattan mobility model performs best but the proposed active inactive mobility model being more realistic in nature performs better than RWPMM and results are very close to the MGMM. It is also concluded that the using Exponential traffic model are better than Poisson traffic model. Finally the results also show that the performance of network using AODV routing protocol is better than OLSR.

\section{FUTURE SCOPE}

In this research paper, AODV and OLSR ad-hoc routing protocols have been used and their performance is analyzed by using the RWPMM, MGM and Active OLSR can additionally be studied for other simulation based mobility models such as Random Walk, Random Direction, Gauss Markov, Column and Reference Point etc. Moreover study can be conducted in area of heterogeneous mobile nodes by using active inactive mobility model.

\section{ACKNOWLEDGMENT}

The authors wish to thank the reviewers and editors for their valuable suggestions and expert comments that help improve the contents of paper.

\section{REFERENCES}

[1] Saadi, Youssef, et al. "Simulation analysis of routing protocols using manhattan grid mobility model in MANET." arXiv preprint arXiv:1304.2035(2013).

[2] Vijayavani, G. R., and G. Prema. "Performance comparison of MANET routing protocols with mobility model derived based on realistic mobility pattern of mobile nodes." Advanced Communication Control and Computing Technologies (ICACCCT), 2012 IEEE International Conference on. IEEE, 2012.

[3] Tayal, Sandeep, and Malay Ranjan Tripathy. "VANET-challenges in selection of vehicular mobility model." Advanced Computing \& Communication Technologies (ACCT), 2012 Second International Conference on. IEEE, 2012

[4] Rohankar, Rashmi, et al. "Performance analysis of various routing protocols (proactive and reactive) for random mobility models of Adhoc networks."Recent Advances in Information Technology (RAIT), 2012 1st International Conference on. IEEE, 2012.

[5] Du, Zhenguo, Peilin Hong, and Kaiping Xue. "Hotspot gravitation mobility model." Communications Letters, IEEE 16.2 (2012): 193 195.

[6] A. Laouiti, A.Qayyum, L.Viennot, "Optimized Link State Routing Protocol For Ad Hoc Networks".

[7] Gong, Zhenhua, and Martin Haenggi. "Interference and outage in mobile random networks: Expectation, distribution, and correlation." Mobile Computing, IEEE Transactions on 13.2 (2014): 337-349

[8] Kuldeep Vats et al, "Simulation and performance Analysis of OLSR, GRP, DSR Routing Protocol using OPNET" IJETED Volume 2, Issue 2, March 2012

[9] T. Kunz and E. Cheng, "On-demand multicasting in ad-hoc networks: Comparing AODV and ODMRP,"pp. 453-454, 2002

[10] Gagangeet Singh Aujla, Sandeep Singh Kang, "Comprehensive Evaluation of AODV, DSR, GRP, OLSR and TORA Routing Protocols with varying number of nodes and traffic applications over MANETs" (IOSR-JCE)

[11] OPNET official website, http://www.opnet.com.

[12] Aamar Nath Muraw et al "performance evaluation of MANET routing protocols GRP, DSR And AODV based on packet size" IJEST vol. 4 no.06 June 2012

[13] C. Perkins, E. Belding-Royer, S. Das, quet, "Ad hoc On- Demand Distance Vector (AODV) Routing”, RFC 3561, July 2003.

[14] Fahim Maan and Nauman Mazhar, "MANET Routing Protocols vs. Mobility Models: A Performance Evaluation", Proc. of IEEEICUFN 2011.

[15] I.S. Hammodi et al, "A comprehensive performance studymof OPNET Modeler for ZigBee WSN" 3rd International conference on Next Generation Mobile Applications, 2009.

16] Mohit Kumar Rashmi Mishra "An Overview of MANET: History, Challenges and Applications" International Journal of Engineering Research and Technology Vol. 3 No.1 Feb-Mar 2012.

[17] Sunil kumar singh,rajesh duvvuru, amit bhattcharjee, "performance evaluation of proative, reactive and hybrid routing protocols with mobility model in manets" IJRET:eissn:239-1163/pissn:2321-7308.

[18] A.pal , jyoti p.singh, p.dutta, " the effect of speed variations on different traffic patterns in mobile ad hoc network", c3it-2012.

[19] Pankaj rohal, ruchika dhaiya and prashant dhaiya, "study and analysis of throughput, delay and packet delivery ratio in manet for topology based routing protocols (AODV, DSR, DSDV)'"IJARET vol.1, issue iii. 
INTERNATIONAL JOURNAL OF INNOVATIVE RESEARCH IN ELECTRICAL, ELECTRONICS, INSTRUMENTATION AND CONTROL ENGINEERING Vol. 3, Issue 10, October 2015

[20] Anjali, Maninder Singh, " simulation and performance analysis of AODV, OLSR, GRP routing protocol by considering IEEE 802.11 standard" IJARCSSE, vol 2, issue 6.

[21] Anuj K. Gupta, Harsh Sadawarti, Anil K. Verma, "performance analysis of manet routing protocols in different mobility models" IJITCS.

[22] Mihail.sichitiu, "mobility models for ad-hoc network".

[23] Nesargi and r. prakash, "distributed wireless channel allocation in network with mobile base station. IEEE conference on computer communication (INFOCOM'99), 1999

[24] Harmanpreet kaur er. Jaswinder singh, “ performance comparision of OLSR, GRP and TORA using OPNET", international journal of advanced research in computer science and software engineering, volume 2, issue 10, October 2012.

[25] Dimitra kampitaki, anastasios a. economides, "simulation study of manet routing protocols under FTP traffic", procedia technology17 (2014)231-238 